\title{
Expression of non-TLR pattern recognition receptors in the spleen of BALB/c mice infected with Plasmodium yoelii and Plasmodium chabaudi chabaudi AS
}

\author{
Anna Rosanas-Urgell ${ }^{1 /+}$, Lorena Martin-Jaular², Julio Ricarte-Filho ${ }^{3}$, \\ Mireia Ferrer ${ }^{2}$, Susana Kalko ${ }^{4}$, Edna Kimura3 ${ }^{3}$, Hernando A del Portillo ${ }^{1,2,5}$ \\ 'Departamento de Parasitologia ${ }^{3}$ Departamento de Biologia Celular, Instituto de Ciências Biomédicas, \\ Universidade de São Paulo, São Paulo, SP, Brasil ${ }^{2}$ Barcelona Centre for International Health Research, \\ Hospital Clínic-Universitat de Barcelona, Barcelona, Spain ${ }^{4}$ Bioinformatics Unit, Institut d'Investigacions Biomèdiques \\ August Pi i Sunyer, Hospital Clinic, Barcelona, Spain ${ }^{5}$ Institució Catalana de Recerca i Estudis Avançats, Barcelona, Spain
}

The spleen plays a crucial role in the development of immunity to malaria, but the role of pattern recognition receptors (PRRs) in splenic effector cells during malaria infection is poorly understood. In the present study, we analysed the expression of selected PRRs in splenic effector cells from BALB/c mice infected with the lethal and nonlethal Plasmodium yoelii strains $17 X L$ and $17 X$, respectively, and the non-lethal Plasmodium chabaudi chabaudi $A S$ strain. The results of these experiments showed fewer significant changes in the expression of PRRs in ASinfected mice than in $17 X$ and $17 X L$-infected mice. Mannose receptor C type 2 (MRC2) expression increased with parasitemia, whereas Toll-like receptors and sialoadhesin (Sn) decreased in mice infected with P. chabaudi AS. In contrast, MRC type 1 (MRCl), MRC2 and EGF-like module containing mucin-like hormone receptor-like sequence 1 (F4/80) expression decreased with parasitemia in mice infected with $17 X$, whereas MRC1 an MRC2 increased and F4/80 decreased in mice infected with 17XL. Furthermore, macrophage receptor with collagenous structure and CD68 declined rapidly after initial parasitemia. SIGNR1 and Sn expression demonstrated minor variations in the spleens of mice infected with either strain. Notably, macrophage scavenger receptor (Msrl) and dendritic cellassociated C-type lectin 2 expression increased at both the transcript and protein levels in 17XL-infected mice with $50 \%$ parasitemia. Furthermore, the increased lethality of $17 \mathrm{X}$ infection in Msr1 -/- mice demonstrated a protective role for Msr1. Our results suggest a dual role for these receptors in parasite clearance and protection in $17 X$ infection and lethality in $17 X L$ infection.

Key words: malaria - spleen - macrophages - receptors - pattern recognition

Malaria remains the most devastating parasitic disease in the world: 100-300 million cases are detected annually and approximately one million malaria-related deaths occur each year in children less than five years old (who.int). Clinical studies and experimental rodent models have demonstrated the importance of the spleen in the development of immunity to malaria (Buffet et al. 2009). Indeed, the spleen removes infected red blood cells (iR$\mathrm{BCs})$ from the circulation, acts as a major erythropoietic and haematopoietic site during embryogenesis and generates immune responses to pathogens (Engwerda et al. 2005, Urban et al. 2005a). In this way, splenic effector cells, which have the capacity to become rapidly activated to produce large amounts of pro-inflammatory cytok-

Financial support: CNPq (to ARU), FAPESP (01/09401-0), European Community's Seventh Framework Programme (FP7/2007-2013 242095), Spanish Ministry of Science and Innovation (SAF2009-07760) + Corresponding author: anna.rosanas@gmail.com

AR-U current address: Papua New Guinea Insitute of Medical Research, Madang 511, Papua New Guinea

Received 1 December 2010

Accepted 15 February 2012 ines, represent the first line of innate immune defence. Further, splenic cells phagocytose infected erythrocytes and help shape downstream adaptive immune responses (Stevenson \& Riley 2004a, Urban et al. 2005b).

Macrophage activation is largely dependent on the interaction of microbial molecules or pathogen-associated molecular patterns (PAMPs) with Toll-like receptors (TLRs) and other pattern recognition receptors (PRRs) (Schofield \& Hackett 1993, Tachado et al. 1996, Naik et al. 2000, Vijaykumar et al. 2001, Zhu et al. 2005, Areschoug \& Gordon 2009). A great deal of research has focused on the role of TLRs in pathogen recognition (Krishegowda et al. 2005); however, the spleen is also rich in macrophage populations that express different sets of PRRs, reflecting the functional specialisation in splenic microenvironments under steady state conditions (Taylor et al. 2005).

Histopathological and physiological studies of the spleen during experimental infections of BALB/c mice with the reticulocyte-prone non-lethal Plasmodium yoelii $17 \mathrm{X}$ strain have revealed that major remodelling of this organ occurs through the creation of a blood-spleen barrier of fibroblastic origin. Infected reticulocytes then adhere to this barrier to avoid clearance (Weiss et al. 1986, Martin-Jaular et al. 2011). Strikingly, this remodelling and the formation of the barrier are not observed 
when mice are infected with the normocyte-prone lethal P. yoelii 17XL strain (Weiss 1989). In the present study, we hypothesised that this unique remodelling of the spleen is accompanied by differential activation of splenic effector cell PRRs throughout the development of lethal and non-lethal Plasmodium infections. To test this hypothesis, we compared the transcript abundance of spleen PRRs during experimental infections of BALB/c mice with $P$. yoelii $17 \mathrm{X}$ and 17XL strains. In addition, to demonstrate the specificity of these results, we also compared the transcript abundance of these same PRRs in BALB/c mice infected with Plasmodium chabaudi chabaudi AS, a model in which mature parasite stages is sequestered from the circulation.

Mice and parasites - Female BALB/c mice were bred in a pathogen-free unit at the Institute of Biomedicine (São Paulo) in accordance with protocols approved by the Ethical Committee of Colégio Brasileiro de Experimentação Animal and the Comissão de Ética em Experimentação Animal (protocol 209/02). All mice were conventionally housed for experimental use and provided with sterile bedding, food and water. For experimental infections with $P$. chabaudi chabaudi AS and $P$. yoelii $17 \mathrm{X}$ and $17 \mathrm{XL}$, the mice were routinely injected intraperitoneally (i.p.) with $10^{6}$ iRBCs. Parasitaemia was monitored on a daily basis between days 2 and 20 post-infection (Supplementary data) by thin tail blood smears and Giemsa staining and the proportion of iRBCs among total RBCs was calculated (Langhorne \& Simon 1989).

For transcript analysis, groups of BALB/c mice were infected with $P$. chabaudi chabaudi AS $(\mathrm{n}=6)$ or $P$. yoelii 17XL $(\mathrm{n}=6)$ or $17 \mathrm{X}(\mathrm{n}=6)$ and two mice of each strain were sacrificed when peripheral parasitaemias reached $1 \%, 10 \%$ and $50 \%$. The experiment was performed twice (biological replicates), resulting in a $n$ of 4 in each experimental group.

Macrophage scavenger receptor (Msrl) knockout (strain B6.Cg-Msr $1^{t m I C s k} / \mathrm{J}$ ) and C57BL/6J wild-type female mice (The Jackson Laboratory, Bar Harbor, Maine, USA) were maintained with water and food ad libitum in the animal facilities of the Hospital Clinic, Barcelona. At 16-18 weeks of age, four-six animals of each group were injected i.p. with $10^{6}$ parasites of either a lethal (17XL) or a non-lethal (17X) strain of $P$. yoelii. All of the experiments were performed according to the guidelines approved by the Ethical Committee of Animal Experimentation of the University of Barcelona.

Tissue preparation - Spleens from P. yoelii 17X and $17 \mathrm{XL}$ and $P$. chabaudi chabaudi AS infected mice were removed under aseptic conditions. To isolate circulating monocytes from the spleen, the mice were anaesthetised with 33\% chloral hydrate solution and infected spleens were removed after perfusion with $0.9 \%$ saline solution and snap-frozen in liquid nitrogen or processed for flow cytometry analysis.

RNA extraction and cDNA synthesis for real-time polymerade chain reaction (RT-PCR) analysis - RNA was extracted from $50 \mathrm{mg}$ total splenic tissue samples with Trizol reagent (Invitrogen). The samples were treated with DNase I (Invitrogen) and reverse transcription (First-Strand cDNA Synthesis, Invitrogen) was performed according to the manufacturer's instructions.

Quantification of macrophage receptor transcript abundance - We compared the expression profiles of different PRRs - mannose receptor C type 1 (MRC1), MRC type 2 (MRC2), Msrl, macrophage receptor with collagenous structure (MARCO), dendritic cell-associated C-type lectin 2 (Dectin2), EGF-like module containing mucin-like hormone receptor-like sequence 1 (F4/80), SIGNR1, sialoadhesin (Sn) and CD68) - with specific primers designed with Primer Express 3.0 software (Supplementary data). The samples were analysed in triplicate and the experiments were repeated twice. cDNA from non-infected mice (calibrator) was included in all PCR experiments. The relative quantification of transcript abundance was performed with SYBR green PCR master mix (Applied Biosystems) using an Applied Biosystems 7500 thermal cycler system. Actin was used as the endogenous control and non-infected mouse transcripts were used as the calibrator.

Data analysis - The relative expression level of each target gene was calculated from triplicate samples analysed in two independent experiments using $\Delta \Delta C_{T}$ method and normalised to the endogenous control. Fold changes were determined by comparing the relative expression levels in infected mice to those in the calibrator (noninfected BALB/c mice) (Livak et al. 2008). Data were analysed using GraphPad Prism software. Statistical significance was determined using two-way ANOVA and differences in parasitaemias in the same strain were evaluated by Bonferroni's post-test.

Flow cytometry analysis - Splenocytes were prepared from the spleens of mice infected with $17 \mathrm{X}$ and $17 \mathrm{XL}$ parasites when the mice reached $1 \%, 10 \%$ and $50 \%$ parasitaemias. The spleens were homogenised and passed through nylon mesh. Spleen cells were preincubated with Seroblock anti-Fc receptor antibody (Abcam, dilution 1/100) for $10 \mathrm{~min}$ and then incubated with an APC-conjugated antibody specific for murine Msrl and a fluorescein isothiocyanate conjugated antibody specific for murine Dectin 2 at $4^{\circ} \mathrm{C}$ for $1 \mathrm{~h}$ and washed twice. 500,000 events were collected using a FACSCanto flow cytometer (BD Biosciences). The data were analysed using either one-way ANOVA with Dunnet's post hoc test or Student's $t$-test with GraphPad Prism software.

Microarray global expression analysis - Total RNA from spleens of mice infected with 17XL or 17X parasite was labelled using an Agilent Low RNA Input Fluorescent Linear Amplification Kit. mRNA from the spleens of each 17X or 17XL infected mouse was labelled with $\mathrm{Cy} 3$ or $\mathrm{Cy} 5$, respectively. Dual hybridisation was performed using the Agilent Whole Mouse Genome microarray according to the manufacturer's protocol. Microarray images were obtained using the GenePix 4000B scanner. Analysis of overrepresentation of functional terms had been done with GOstats tool, which finds statistically overrepresented gene ontologies within a group of genes. The program determined the annotated 
GO terms and the GO terms that are associated (i.e., in the path) with genes analysed. The number of appearances of each GO term was then counted. Fisher's exact test was performed to determine whether the observed difference were significant. This resulted in a p-value for each GO category that was used to determine whether the observed counts could have occurred by chance. All data were deposited at GEO under accession GSE17603.

The expression profile of a variety of splenic effector cell PRRs was assessed in samples of total spleen tissue from BALB/c mice experimentally infected with the P. yoelii 17X and 17XL strains. Because of differences in the growth of these parasites, the analysis was performed when the mice reached $1 \%, 10 \%$ and $50 \%$ peripheral parasitaemia. To determine the specificity of the changes observed, the expression of these PRRs was also analysed in BALB/c mice infected with (the normocyte-prone non-lethal) $P$. chabaudi chabaudi AS strain, which in contrast to $P$. yoelii, sequesters in the deep capillaries of internal organs (Cox et al. 1987). Overall, the results revealed more dramatic increases in the expression of PRRs in mice infected with 17XL than in mice infected with 17X (Fig. 1).

Expression of most of the PRRs was lower in the spleens of $P$. chabaudi chabaudi AS-infected mice than in $P$. yoelii-infected mice, but these changes were not statistically significant (Fig. 1). These data support previous studies showing that $P$. chabaudi chabaudi AS is not cleared by the spleen due to its adhesion to internal organs. In contrast, the expression levels of most of the PRRs were significantly different in P. yoelii $17 \mathrm{X}$ and 17XL infected mice (Fig. 1).

The expression of the macrophage receptors MRC1 and MRC2 was decreased in the $17 \mathrm{X}$-infected mice with $10 \%$ parasitaemia $(\mathrm{p}<0.001)$, but the expression of both of these receptors was increased in 17XL-infected mice with the same degree of parasitaemia $(p<0.001)$. Similarly, the expression level of the F4/80 receptor in mice infected with the $17 \mathrm{X}$ strain was increased when the mice reached $1 \%$ parasitaemia (11.3-fold). This increased parasitaemia was maintained in mice with $10 \%$ parasitaemia (12.5-fold, $\mathrm{p}>0.05)$ but decreased in mice with $50 \%$ parasitaemia (5.9-fold, $p<0.001)$. The expression of the three receptors fluctuated less than five-fold over the course of P. chabaudi chabaudi AS infection, with the exception of a slight increase in MRC2 expression when parasitaemia peaked at $50 \%(\mathrm{p}<0.05)$. Interestingly, $\mathrm{MRC1} / 2$ - and F4/80 - expressing macrophages are predominantly located in the red pulp of the spleen in the absence of infection (Taylor et al. 2005). We observed that $10 \%$ parasitaemia was achieved at approximately day 6 post-infection in mice infected with the 17X strain (Supplementary data).

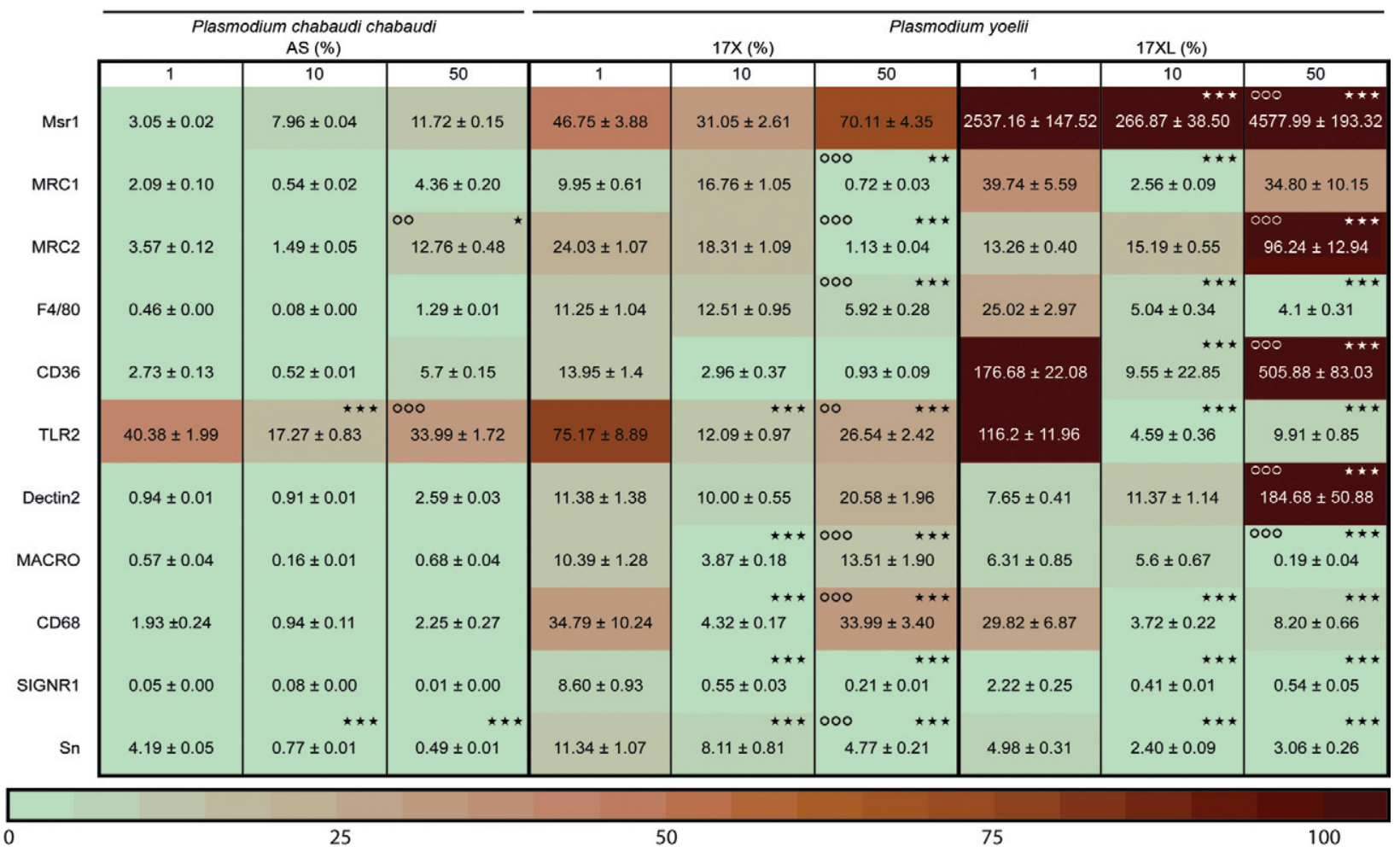

Fig. 1: the heat map represents colour-coded relative quantification values for expression levels (x-fold changes compared to non-infected control) of splenic effector cell pattern recognition receptors (PRRs) between P. yoelii 17X, 17XL and P. chabaudi chabaudi AS at 1\%, 10\% and 50\% parasitaemias ( $n=4$ mice/group). Additionally, values for mean and standard deviation are included in the boxes and data was pooled from two independent experiments. Data was evaluated by a two-way ANOVA test of variance and Bonferroni post-test, vs. $1 \%$ infection $(*$ : $\mathrm{p}<0.05 ; * *$ : $\mathrm{p}<0.01 ; * *: \mathrm{p}<0.001)$ and $10 \%$ infection $\left({ }^{\circ o}: \mathrm{p}<0.01\right.$; $\left.{ }^{\circ o o}: \mathrm{p}<0.001\right)$. Dectin: dendritic cell-associated C-type lectin; MARCO: macrophage receptor with collagenous structure; MRC: mannose receptor C; Msr: macrophage scavenger receptor; Sn: sialoadhesin; TLR: Toll-like receptor. 
A recent study has reported that a blood-spleen barrier of fibroblastic origin forms during the initial days of $17 \mathrm{X}$ infection in $\mathrm{BALB} / \mathrm{c}$ mice and prevents parasite contact with macrophages (Martin-Jaular et al. 2011). It is tempting to speculate that newly formed tissue barrier cells partially conceal $17 \mathrm{X}$ iRBCs from effector cells and, as a consequence, the expression of these receptors may be down-regulated at this level of parasitaemia.

Although statistically significant $(\mathrm{p}<0.001)$ fluctuations in the expression levels of SIGNR1, Sn, MARCO and CD68 were observed, the low expression levels of these receptors were generally stable in mice infected with both strains of $P$. yoelii. Similar results were observed in biological replicates. These results suggest that these receptors are not likely to be involved in the process of innate immune activation initiated by malaria infection.

The analysis of more widely expressed PRRs showed that TLR 2 was highly expressed in all strains at $1 \%$ parasitaemia (40.4-fold, 75.2-fold and 116.2-fold increases in mice with for $P$. chabaudi chabaudi, AS, 17X and 17XL, respectively) and its expression decreased with increasing parasitaemia. These data are in agreement with the perceived role of TLR2 in initiating host defence upon detection of PAMPs (Erdman et al. 2008). Likewise, following infection with each of the strains, the expression of CD36 increased when the mice reached 1\% parasitaemia (increases of 2.7-fold, 14-fold and 176.7-fold for P. chabaudi chabaudi AS, 17X and 17XL, respectively); however, CD36 expression decreased when the mice reached $10 \%$ parasitaemia (decreases of 0.5 -fold, 3-fold and 10-fold for P. chabaudi chabaudi AS, 17X and $17 \mathrm{XL}$, respectively). CD36 expression levels in mice with $50 \%$ parasitaemia varied among the mice infected with different species (5.7-fold for P. chabaudi chabau$d i$, no change for 17X and 505.9-fold for 17XL). Previous studies have shown that TLR2 is activated by Plasmodium falciparum glycosylphosphatidylinositol in both human and murine macrophages, resulting in the production of tumour necrosis factor (TNF)- $\alpha$, which appears to play a dual role in malaria infection. Some reports have suggested its importance in controlling parasites, while others have proposed a pathogenic role (Richards 1997, Krishnegowda et al. 2005, Zhu et al. 2011). In addition a role in mediating non-opsonic phagocytosis of iRBCs by macrophages and monocytes (McGilvray et al. 2000, Serghides \& Kain 2002), CD36 has been reported to enhance TLR2 signalling by acting as a coreceptor for TLR2 in the induction of TNF- $\alpha$ (Patel et al. 2007, Erdman et al. 2009). Here, we observed a 177 -fold increase in CD36 expression in mice infected with the 17XL strain with $1 \%$ parasitaemia.

Dectin2 has been shown to be involved in the recognition of several pathogens (McGreal et al. 2006, Sato et al. 2006, Robinson et al. 2009). Analysis of the expression of this receptor in mice infected with $17 \mathrm{X}$ revealed an increase in Dectin2 expression in mice with $1 \%$ parasitaemia. The expression level of Dectin 2 remained steady in mice with $10 \%$ parasitaemia $(\mathrm{p}>0.05)$ and further increased in mice with $50 \%$ parasitaemia. In contrast, expression of Dectin2 was highly correlated with increasing parasitaemia in mice infected with the $17 \mathrm{XL}$ strain $\left(r^{2}=0.988\right)$. Although Dectin2 has been shown to be involved in the recognition of several pathogens, to our knowledge, this is the first time its expression has been described in malaria.

Although Msr1 -/- mice have been used for experimental infection studies, the role of Msrl during malaria infection remains unclear and different groups have reported conflicting data (Nogami et al. 1998, CunhaRodrigues et al. 2006, Inoue et al. 2006). In our analysis, the expression of Msrl was observed without significant changes in mice with $1 \%$ and $10 \%$ parasitaemia (increase of 46.8-fold and 31.1-fold respectively), but expression was much higher in mice with $50 \%$ parasitaemia (70.1fold) following infection with the 17X strain. In addition, the expression of Msrl was high throughout the course of $17 \mathrm{XL}$ infection, with peak expression at $50 \%$ parasitaemia (increase of 4.577-fold, $\mathrm{p}<0.01$ ). In contrast, expression of Msrl in mice infected with $P$. chabaudi chabaudi AS reached a maximum level that was only 12 -fold higher than that in uninfected mice. Moreover, transcriptional analyses of spleens from $17 \mathrm{X}$ and $17 \mathrm{XL}-$ infected mice with $1 \%, 10 \%$ and $50 \%$ parasitaemia using commercially available arrays identified this gene as one of the most variable (Supplementary data).

We next investigated whether Dectin2 and Msrl protein levels also changed. To this end, BALB/c mice were infected with $P$. yoelii 17X and 17XL strains. Splenocytes were then obtained from animals with $1 \%, 10 \%$ and $50 \%$ parasitaemias, stained with antibodies specific for different receptors and analysed by flow cytometry. The number of cells expressing each PPR is shown as percentages of the total splenocyte population (Fig. 2). Phenotypic analysis of splenocytes revealed that the levels of Msr1 and Dectin 2 proteins were also altered during infections; however, the changes in protein levels were not always positively correlated with changes in transcript levels (Fig. 2). The differences observed between transcript
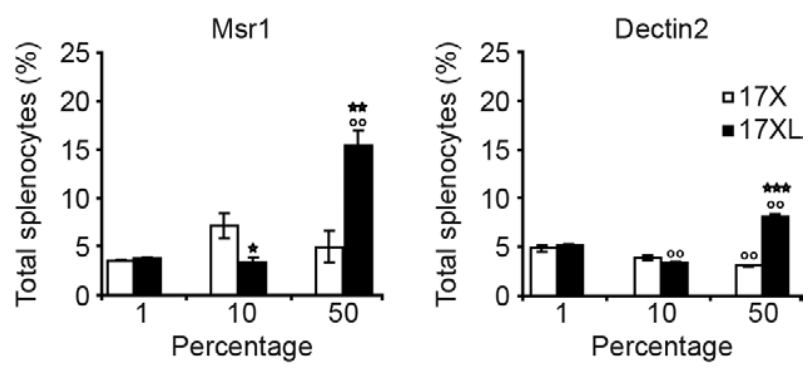

Fig. 2: flow cytometry analysis of splenocytes stained for macrophage scavenger receptor (Msr)1 and dendritic cell-associated C-type lectin (Dectin)2. Splenocytes of mice infected with Plasmodium yoelii $17 \mathrm{X}$ and $17 \mathrm{XL}$ parasites at $1 \%, 10 \%$ and $50 \%$ parasitaemias were labelled with conjugated antibody and analysed in a FACSCanto flow cytometer. Mean percentages \pm standard error of the mean (SEM) of total splenocytes are shown from three mice per group. Percentages of $\mathrm{Msr}^{+}$and Dectin2 $2^{+}$cells were $1.41 \pm 0.14$ and $2.79 \pm 0.26$ for control animals $(n=3)$. Data was evaluated by analysis of variance vs. $1 \%$ infection for each strain separatelly $\left({ }^{\circ}: \mathrm{p}<0.05 ;{ }^{\circ}: \mathrm{p}<0.01\right)$ (Dunnet post hoc test). Differences between 17X and 17XL strain were assesed using Student's $t$-test $(*: \mathrm{p}<0.05 ; * *: \mathrm{p}<0.01 ; * * *$ : $<0.001)$. 
and protein levels in mice with the same levels of parasitaemia may be due to either methodological constraints, such as RT-PCR values relative to the non-infected control, or differences in absolute numbers of splenocytes, or other variables, such as post-transcriptional regulation. The data obtained from the flow cytometry experiments, however, revealed changes in the levels of PRRs proteins and thus support a dual role of these receptors in parasite clearance and protection during $17 \mathrm{X}$ infection and in lethality during $17 \mathrm{XL}$ infection.

In order to further explore the role of the Msrl receptor during malaria-induced innate immune responses in the spleen, we infected Msr1 -/- and C57BL/6J wild-type female mice with $17 \mathrm{XL}$ or $17 \mathrm{X}$. We were unable to perform these experiments in Dectin2 -/- mice because they are not commercially available. Survival curves revealed a significant increase in lethality in Msrl-deficient mice infected with 17X (log-rank test $\mathrm{p}<0.01)$. In contrast, Msrl-deficient mice appeared to survive longer than wild-type mice following 17XL-infection; however, this trend was not statistically significant (log-rank test $p$ $=0.06$ ) (Fig. 3). These findings suggest that Msrl plays an important protective role during $17 \mathrm{X}$ infections that require increased expression at the transcript and protein levels (Figs 1, 2). In contrast, hyperactivation and uncontrolled immune responses during 17XL infections ultimately lead to the death of the host.

Severe malaria syndromes are thought to be due at least in part to the overproduction of pro-inflammatory cytokines by cells of the innate immune system (Stevenson \& Riley 2004, Schofield \& Grau 2005, Urban et al. 2005b, Clark et al. 2006, Bakir et al. 2011). Consistent with a role for such responses in the pathogenesis of sepsis and other hyperacute inflammatory states, there also appears to be a fine line between the beneficial role of inflammatory responses and the pathogenic side-effects that can result from immune hyperactivation (Clark et al. 2006). Accordingly, it has been postulated that in mild malaria the pro-inflammatory response may be effectively down-regulated, whereas inflammation may remain unbalanced and uncontrolled in severe and fatal malaria cases (Malaguarnera \& Musumeci 2002). Interestingly, analysis of the 10 most variables genes revealed changes in different functional pathways between mice infected with the 17X and 17XL strains: endocytosis

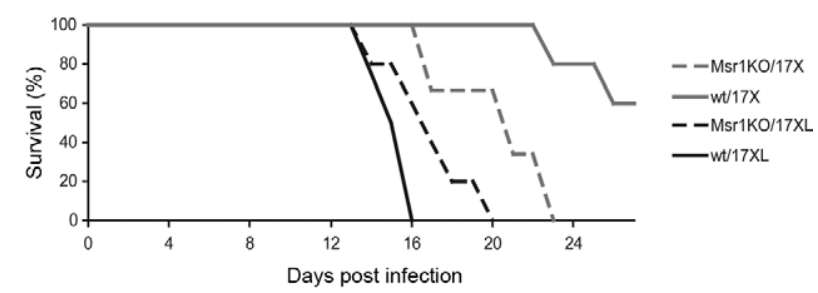

Fig. 3: survival curve of macrophage scavenger receptor (Msr)1 knockout (KO) and C57BL/6J wild-type (wt) female mice infected with 17XL or 17X strain of Plasmodium yoelii. Results correspond to four-six animals per group. Differences among Msrl -/- and wt mice infected with the same strain were evaluated by long-Rank test. and phosphate transport were most significantly affected in 17X-infected mice and cell death and apoptosis were most significantly affected in 17XL-infected mice (Supplementary data). Remarkably, Dectin2 and Msr1 expression was increased at the transcript and protein levels in 17XL-infected mice compared to $17 \mathrm{X}$-infected mice with $50 \%$ parasitaemia. These differences in expression ultimately resulted in parasite clearance and recovery in 17X-infected mice and elevated parasitaemia and death in 17XL-infected mice strain. Therefore, our results suggest that Dectin2 and Msrl play dual roles in the development of innate inflammatory responses. Both PRRs seem to be involved in pathogenic responses in 17XL-infected mice, whereas they play a role in the protective response in 17X-infected mice. These data also indirectly support the previous observation that a cellular barrier forms in spleen during non-lethal Plasmodium infection (Weiss et al. 1986, Martin-Jaular et al. 2011) and suggest that this barrier prevents hyperactivation of pro-inflammatory responses that lead to pathogenic effects and eventually the death of the host.

\section{ACKNOWLEDGEMENTS}

To the anonymous reviewer, whose suggestions have improved significantly the quality of this paper, to Dr Leanne Robinson, for her critical review, to Dr Sergio Rodriguez, for discussions, and for the training in handling infections of $\mathrm{BALB} / \mathrm{c}$ mice and rodent malaria species and Marcio Massao Yamamoto, for technical assitance.

\section{REFERENCES}

Areschoug T, Gordon S 2009. Scavenger receptors: role in innate immunity and microbial pathogenesis. Cell Microbiol 11: 1160-1169.

Bakir HY, Tomiyama C, Abo T 2011. Cytokine profile of murine malaria: stage-related production of inflammatory and anti-inflammatory cytokines. Biomed Res 32: 203-208.

Buffet PA, Safeukui I, Milon G, Mercereau-Puijalon O, David PH 2009. Retention of erythrocytes in the spleen: a double-edged process in human malaria. Curr Opin Hematol 16: 157-164.

Clark IA, Budd AC, Alleva LM, Cowden WB 2006. Human malarial disease: a consequence of inflammatory cytokine release. Malar J 5: 85 .

Cox J, Semoff S, Hommel M 1987. Plasmodium chabaudi: a rodent malaria model for in-vivo and in-vitro cytoadherence of malaria parasites in the absence of knobs. Parasite Immunol 9: 543-561.

Cunha-Rodrigues M, Portugal S, Febbraio M, Mota MM 2006. Infection by and protective immune responses against Plasmodium berghei ANKA are not affected in macrophage scavenger receptors A deficient mice. BMC Microbiol 6: 73.

Engwerda CR, Beattie L, Amante FH 2005. The importance of the spleen in malaria. Trends Parasitol 21:75-80.

Erdman LK, Cosio G, Helmers AJ, Gowda DC, Grinstein S, Kain KC 2009. CD36 and TLR interactions in inflammation and phagocytosis: implications for malaria. J Immunol 183: 6452-6459.

Erdman LK, Finney CA, Liles WC, Kain KC 2008. Inflammatory pathways in malaria infection: TLRs share the stage with other components of innate immunity. Mol Biochem Parasitol 162: 105-111.

Inoue M, Xuan X, Fujisaki K, Igarashi I, Suzuki H 2006. Short report: role of type I/II scavenger receptors in malarial infection in C57BL/6J mice. Am J Trop Med Hyg 75: 178-181. 
Krishnegowda G, Hajjar AM, Zhu J, Douglass EJ, Uematsu S, Akira S, Woods SA, Gowda DC 2005. Induction of proinflammatory responses in macrophages by the glycosylphosphatidylinositols of Plasmodium falciparum. J Biol Chem 280: 8606-8616.

Langhorne J, Simon B 1989. Limiting dilution analysis of the T cell response to Plasmodium chabaudi chabaudi in mice. Parasite Immunol 11: 545-559.

Malaguarnera L, Musumeci M 2002. The immune response to Plasmodium falciparum malaria. Lancet 2: 472-478.

Martin-Jaular L, Ferrer M, Calvo M, Rosanas-Urgell A, Kalko S, Graewe S, Soria G, Cortadellas N, Ordi J, Planas A, Burns J, Heussler V, del Portillo HA 2011. Strain-specific spleen remodelling in Plasmodium yoelii infections in BALB/c mice facilitates adherence and spleen macrophage-clearance escape. Cell Microbiol 13: 109-122.

McGilvray ID, Serghides L, Kapus A, Rotstein OD, Kain KC 2000. Nonopsonic monocyte/macrophage phagocytosis of Plasmodium falciparum-parasitized erythrocytes: a role for CD36 in malarial clearance. Blood 96: 3231-3240.

McGreal EP, Rosas M, Brown GD, Zamze S, Wong SY, Gordon S, Martinez-Pomares L, Taylor PR 2006. The carbohydrate-recognition domain of Dectin-2 is a C-type lectin with specificity for high mannose. Glycobiology 16: 422-430.

Naik RS, Branch OH, Woods SA, Vijaykumar M, Perkins DJ, Nahlen BL, Lal AA, Cotter RJ, Costello CE, Ockenhouse CF, Davidson EA, Gowda DC 2000. Glycosylphosphatidylinositol anchors of Plasmodium falciparum: molecular characterization and naturally elicited antibody response that may provide immunity to malaria pathogenesis. J Exp Med 192: 1563-1576.

Nogami S, Watanabe J, Nakagaki K, Nakata K, Suzuki H, Fujisawa M, Kodama T, Kojima S 1998. Involvement of macrophage scavenger receptors in protection against murine malaria. Am J Trop Med Hyg 59: 843-845.

Patel SN, Lu Z, Ayi K, Serghides L, Gowda DC, Kain KC 2007. Disruption of CD36 impairs cytokine response to Plasmodium falciparum glycosylphosphatidylinositol and confers susceptibility to severe and fatal malaria in vivo. J Immunol 178: 3954-3961.

Richards AL 1997. Tumour necrosis factor and associated cytokines in the host's response to malaria. Int J Parasitol 27: 1251-1263.

Robinson MJ, Osorio F, Rosas M, Freitas RP, Schweighoffer E, Gross O, Verbeek JS, Ruland J, Tybulewicz V, Brown GD, Moita LF, Taylor PR, Reis e Sousa C 2009. Dectin-2 is a syk-coupled pattern recognition receptor crucial for Th17 responses to fungal infection. $J$ Exp Med 206: 2037-2051.

Sato K, Yang XL, Yudate T, Chung JS, Wu J, Luby-Phelps K, Kimberly RP, Underhill D, Cruz PD Jr, Ariizumi K 2006. Dectin-2 is a pattern recognition receptor for fungi that couples with the $\mathrm{Fc}$ receptor gamma chain to induce innate immune responses. $J$ Biol Chem 281: 38854-38866.

Schofield L, Grau GE 2005. Immunological processes in malaria pathogenesis. Nat Rev Immunol 5: 722-735.

Schofield L, Hackett F 1993. Signal transduction in host cells by a glycosylphosphatidylinositol toxin of malaria parasites. $J$ Exp Med 177: 145-153.

Serghides L, Kain KC 2002. Mechanism of protection induced by vitamin A in falciparum malaria. Lancet 359: 1404-1406.

Stevenson MM, Riley EM 2004. Innate immunity to malaria. Nat Rev Immunol 4: 169-180.

Tachado SD, Gerold P, McConville MJ, Baldwin T, Quilici D, Schwarz RT, Schofield L 1996. Glycosylphosphatidylinositol toxin of Plasmodium induces nitric oxide synthase expression in macrophages and vascular endothelial cells by a protein tyrosine kinase-dependent and protein kinase $\mathrm{C}$-dependent signaling pathway. J Immunol 156: 1897-1907.

Taylor PR, Martinez-Pomares L, Stacey M, Lin HH, Brown GD, Gordon S 2005. Macrophage receptors and immune recognition. Annu Rev Immunol 23: 901-944.

Urban BC, Hien TT, Day NP, Phu NH, Roberts R, Pongponratn E, Jones M, Mai NT, Bethell D, Turner GD, Ferguson D, White NJ, Roberts DJ 2005a. Fatal Plasmodium falciparum malaria causes specific patterns of splenic architectural disorganization. Infect Immun 73: 1986-1994.

Urban BC, Ing R, Stevenson MM 2005b. Early interactions between blood-stage Plasmodium parasites and the immune system. Curr Top Microbiol Immunol 297: 25-70.

Vijaykumar M, Naik RS, Gowda DC 2001. Plasmodium falciparum glycosylphosphatidylinositol-induced TNF-alpha secretion by macrophages is mediated without membrane insertion or endocytosis. J Biol Chem 276: 6909-6912.

Weiss L 1989. Mechanisms of splenic control of murine malaria: cellular reactions of the spleen in lethal (strain 17XL) Plasmodium yoelii malaria in BALB/c mice and the consequences of pre-infective splenectomy. Am J Trop Med Hyg 41: 144-160.

Weiss L, Geduldig U, Weidanz W 1986. Mechanisms of splenic control of murine malaria: reticular cell activation and the development of a blood-spleen barrier. Am J Anat 176: 251-285.

Zhu J, Krishegowda G, Gowda DC 2005. Induction of proinflammatory responses in macrophages by the glycosylphosphatidylinositols of Plasmodium falciparum. J Biol Chem 280: 8617-8627.

Zhu J, Krishnegowda G, Li G, Gowda DC 2011. Proinflammatory responses by glycosylphosphatidylinositols (GPIs) of Plasmodium falciparum are mainly mediated through the recognition of TLR2/TLR1. Exp Parasitol 128: 205-211. 
SYBR green primers targeting different splenic pattern recognition receptors genes designed using Primer Express 3.0 software

\begin{tabular}{|c|c|c|c|}
\hline Receptor & Accession & Forward & Reverse \\
\hline $\mathrm{F} 4 / 80$ & NM_010130 & catctgtggetgectccet & ccttgggagcettctggatc \\
\hline MRC1 & NM_008625 & ctcagcaagcgatgtgcct & gcatagggccaccactgatt \\
\hline MRC2 & NM_008626 & gggaaccagacattggtcga & cctcctgaaagcgtacccac \\
\hline Msr1 & NM_031195 & cctggaggtcgaggaaac & tccegacetcectggc \\
\hline SIGNR1 & NM_126972 & ttcttctccaagtcccagcg & tttgcaagctgtgacggc \\
\hline MARCO & NM_010766 & tgaagatgcgggtgtggaa & tgatgacctctcgggetcc \\
\hline Dectin 2 & NM_020001 & cctcaacaatggtgcaggaa & ttggacactgggacatcgg \\
\hline $\mathrm{Sn}$ & NM_011426 & cetgctcattccetgeatct & ttggacactgggacatcgg \\
\hline CD68 & NM_009853.1 & tggcggtggaatacaatgtg & tgaatgtccactgtgctgcc \\
\hline CD36 & NM_007643 & aaccagtgetctccettgattc & cgatcacagcccattctcet \\
\hline TLR2 & NM_011905 & atccecttcectcacttcca & tctgggcaccagcctagg \\
\hline Actin & NM_007393 & gcgggcgacgatgct & agggcggeccacgat \\
\hline
\end{tabular}

gene specificity of all primers was confirmed using BLAST (ncbi.nlm.nih.gov/BLAST/). Dectin: dendritic cell-associated Ctype lectin; MARCO: macrophage receptor with collagenous structure; MRC: mannose receptor C; Msr: macrophage scavenger receptor; Sn: sialoadhesin; TLR: Toll-like receptor.

Analysis of over-representation of functional terms using the tool Gostats of the 10 most variable genes in lines 17X (Cy3) and 17XL (Cy5) obtained from the microarray expression analysis of the spleen of BALB/c mice infected with Plasmodium yoelii $17 \mathrm{X}$ and $17 \mathrm{XL}$ strain at $1 \%, 10 \%$ and $50 \%$ parasitaemia

\begin{tabular}{lcc}
\hline GO term & $\begin{array}{c}\text { CY3 X p } \\
\text { and genes }\end{array}$ & $\begin{array}{c}\text { CY5 XL p } \\
\text { and genes }\end{array}$ \\
\hline Taxis & 0.00101 & 0.000934 \\
GO: 0042330 & ccl2 ccl5 ifng & ccl2 $\mathrm{ccl}$ ifng \\
Phosphate transport & 0.00841 & - \\
GO:0006817 & marco msrl & 0.00157 \\
Immune response & 0.01 & ccl2 ccl5 ifng ptprc \\
GO:0006955 & ccl2 ccl5 ifng & - \\
Endocytosis & 0.0145 & 0.00778 \\
GO:0006897 & mrcl msrl & ifng tnfrsf18 ptprc \\
Apoptosis & - & 0.0085 \\
GO:0006915 & - & ifng tnfrsf18 ptprc \\
Cell death & &
\end{tabular}

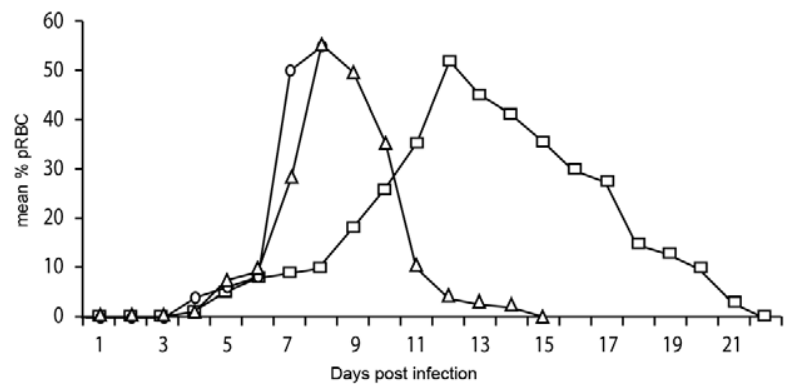

Course of parasitaemia in BALB/c infections with Plasmodium yoelii 17X and 17XL strains and with Plasmodium chabaudii chabaudi AS. Groups of six BALB/c mice were challenged with $1 \times 10^{6}$ P. yoelii 17X (squares), 17XL (circles) and P. chabaudi chabaudi AS (triangles) infected red blood cells (iRBCs) and course of parasitaemia was monitored by enumerating iRBCs in tail blood thin smears stained with Giemsa. 